\title{
Naringenin inhibits alcoholic injury by improving lipid metabolism and reducing apoptosis in zebrafish larvae
}

\author{
HAIYAN LIN ${ }^{1,4^{*}}$, ZHENTING ZHOU $^{1,5^{*}}$, WEICHAO ZHONG $^{4}$, PENG HUANG ${ }^{1}$, NING MA ${ }^{3}$, \\ YUQING ZHANG ${ }^{3}$, CHUYING ZHOU $^{1}$, YULING LAI ${ }^{1}$, SHAOHUI HUANG $^{1}$, \\ HAIYAN AN ${ }^{1}$, XUEGANG SUN ${ }^{1,2}$, LEI GAO ${ }^{1,2}$ and ZHIPING LV ${ }^{1}$
}

\begin{abstract}
${ }^{1}$ School of Traditional Chinese Medicine, Southern Medical University; ${ }^{2}$ The Key Laboratory of Molecular Biology, State Administration of Traditional Chinese Medicine, School of Traditional Chinese Medicine, Southern Medical University; ${ }^{3}$ Key Laboratory of Zebrafish Modeling and Drug Screening for Human Diseases of Guangdong Higher Education Institutes, Department of Developmental Biology, Institute of Genetic Engineering, School of Basic Medical Sciences, Southern Medical University, Guangzhou, Guangdong 510515; ${ }^{4}$ Department of Liver Diseases, Shenzhen Traditional Chinese Medicine Hospital, Shenzhen, Guangdong 518033; ${ }^{5}$ Department of Neurology, Huzhou Traditional Chinese Medicine Hospital, Huzhou, Zhejiang 313000, P.R. China
\end{abstract}

Received February 10, 2017; Accepted July 18, 2017

DOI: $10.3892 / o r .2017 .5965$

\begin{abstract}
Alcoholic liver disease (ALD) includes a spectrum of hepatic abnormalities that range from isolated alcoholic steatosis to steatohepatitis and cirrhosis. Naringenin, a predominant flavanone in grapefruit, increases resistance to oxidative stress and inflammation and protects against multiple organ injury in various animal models. However, the specific mechanisms responsible for protection against alcoholic injury are poorly understood. In the present study,
\end{abstract}

Correspondence to: Professor Zhiping Lv or Dr Lei Gao, School of Traditional Chinese Medicine, Southern Medical University, Guangzhou, Guangdong 510515, P.R. China

E-mail: 1zp48241@126.com

E-mail: raygaolei@smu.edu.cn

*Contributed equally

Abbreviations: ALD, alcoholic liver disease; PBS, phosphatebuffered saline; PFA, paraformaldehyde; H\&E, hematoxylin and eosin; qPCR, real-time quantitative PCR; hpf, h post-fertilization; $\mathrm{dpf}$, days post-fertilization; rpp0, ribosomal protein $\mathrm{P} 0$; cyp2e1, cytochrome $\mathrm{P} 450$ family 2 subfamily $\mathrm{E}$ member 1; cyp2y3, cytochrome P450, family 2, subfamily Y, polypeptide 3; cyp3a65, cytochrome $\mathrm{P} 450$, family 3 , subfamily $\mathrm{A}$, polypeptide 65 ; cyp $3 a$, cytochrome $\mathrm{P} 450$, family 3 , subfamily A; chop, DNA-damageinducible transcript 3; gadd45aa, growth arrest and DNA damage-inducible, $\alpha$, a; hmgcra, HMG coenzyme A reductase a; hmgcrb, HMG coenzyme A reductase b; fasn, fatty acid synthase; fads 2 , fatty acid desaturase 2; fabp10 $\alpha$, fatty acid binding protein 10a; edem1, ER degradation-enhancing $\alpha$-mannosidase-like protein 1; echs1, enoyl-CoA hydratase, short chain 1

Key words: naringenin, zebrafish embryos, alcoholic liver disease, apoptosis, lipid metabolism we aimed to investigate the effect of naringenin on alcoholic events and the molecular regulatory mechanisms of naringenin in the liver and whole body of zebrafish larvae following exposure to $350 \mathrm{mmol} / \mathrm{l}$ ethanol for $32 \mathrm{~h}$. Zebrafish larvae 44 days post-fertilization (dpf); wild-type (WT) and a transgenic line with liver-specific eGFP expression $[\operatorname{Tg}(\mathrm{lfabp} 10 \alpha-e G F P)]\}$ were used to establish an alcoholic fatty liver model in order to evaluate the effects of naringenin treatment on anti-alcoholic injury. Naringenin significantly reduced alcoholic liver morphological phenotypes and the expression of alcohol and lipid metabolism-related genes, including cyp $2 y 3$, cyp $3 a 65$, hmgcra, hmgcrb, fasn, fabpl0a, fads 2 and echsl, in zebrafish larvae. Naringenin also attenuated hepatic apoptosis in larvae as detected by TUNEL staining, consistent with the expression of critical biomarkers of endoplasmic reticulum stress and of DNA damage genes (chop, gadd $45 \alpha a$ and edeml). The present study showed that naringenin inhibited alcohol-induced liver steatosis and injury in zebrafish larvae by reducing apoptosis and DNA damage and by harmonizing alcohol and lipid metabolism.

\section{Introduction}

Hepatic steatosis is an early and common sign of alcohol consumption and possible progression to alcoholic liver disease (ALD), which is a major liver disease and an important health issue in America and worldwide (1). ALD includes a series of phenotypes ranging from simple steatosis to steatohepatitis, progressive fibrosis, cirrhosis and hepatocellular carcinoma (2). The worldwide growing prevalence of ALD suggests that more affordable treatments are needed.

Epidemiological studies have revealed an association between an increased consumption of dietary flavonoids and a reduced risk of dyslipidemia disease (3). The flavonoid naringenin [5,7-dihydroxy-2-(4-hydroxyphenyl)chroman4-one] has been investigated as a possible candidate agent 
to protect against inflammatory injuries (4). Naringenin was found to improve glucose and insulin tolerance, reduce hepatic lipid accumulation, and attenuate dyslipidemia and atherosclerosis in mouse models $(5,6)$. Naringenin induced hepatic fatty acid oxidation, which reduces the availability of lipids, particularly triglycerides, for the assembly and secretion of apolipoprotein B-containing lipoproteins, leading to reduced hepatic lipid accumulation and improved dyslipidemia (6). Naringenin works in a manner similar to that of metformin, a medicine used for type 2 diabetes, by reducing hepatic glucose production in hepatocytes (7). The effects of naringenin on dysregulated metabolism are related to reductions in adipose mass and ectopic lipid deposition in the liver (5). Naringenin modulates the levels of necrotic inflammation, reduces lipid and protein oxidation, recruits the antioxidative defense system and markedly promotes extracellular matrix degradation (8). Additionally, naringenin was found to prevent diet-induced weight gain and adiposity by increasing whole-body energy expenditure (5). Other polyphenolic compounds prevented diet-induced adipose tissue accumulation through a variety of mechanisms, including inhibition of preadipocyte differentiation, lipolysis stimulation and increased mitochondrial function (9). However, the role of naringenin in alcohol-induced hepatic steatosis and its molecular mechanisms remain to be fully elucidated.

Taking into account the above-mentioned findings, the present study was undertaken to investigate the effects of naringenin on alcohol-induced hepatic steatosis injury of zebrafish larvae in vivo. To delineate the mechanisms by which naringenin exerts its effects on alcohol metabolism, antidyslipidemia and hepatocyte damage in ALD, we evaluated several critical genes related to alcohol and lipid metabolism. Morphological examinations of the whole zebrafish body and liver were also performed to further substantiate the beneficial effects of naringenin on pathological alterations induced by alcohol exposure. For the first time, we evaluated the mechanisms by which naringenin regulates alcohol and lipid homeostasis in zebrafish larvae, and we conclude that naringenin can protect against alcohol-induced metabolic dysregulation. Collectively, these findings demonstrated that naringenin had marked lipid-lowering potential, could normalize alcohol and lipid metabolism and could prevent hepatic steatosis. The ability of naringenin to modulate metabolic pathways linked to ALD suggests that citrus flavonoids represent valuable tools in the search for regulators of alcohol metabolism, lipid homeostasis and liver damage.

\section{Materials and methods}

Animal husbandry and treatments. Adult wild-type zebrafish (WT, AB strain) and a liver-specific eGFP expression transgenic zebrafish line, $T g(l f a b p 10 \alpha-e G F P)$, were maintained on a $14: 10 \mathrm{~h}$ light:dark cycle at $28^{\circ} \mathrm{C}$. Embryos were collected following natural spawning and raised at $28^{\circ} \mathrm{C}$. All zebrafish protocols were approved by the Institutional Animal Care and Use Committee of Southern Medical University.

Larvae were exposed to $350 \mathrm{mmol} / 1$ ethanol $(2 \% \mathrm{EtOH})$ in fish water starting at $96-98 \mathrm{~h}$ post fertilization (hpf) for up to $32 \mathrm{~h}$ as previously described (10). Zebrafish larvae in the model group were randomly divided into 5 groups and placed in 6-well plates. We dissolved monomers of naringenin in dimethyl sulfoxide (DMSO) and diluted the stock solution with fish water to obtain different concentrations. All groups were given $8 \mathrm{ml}$ of water, and we added appropriate naringenin concentrations to the drug-treated groups and $0.1 \%$ DMSO (by volume concentration) to the DMSO group. The 6-well plates were placed in an incubator for $48 \mathrm{~h}$; we then observed and recorded the general situation of each group.

Oil Red $O$ staining. Oil Red O staining was carried out as previously described (11). Zebrafish larvae were collected in $1.5 \mathrm{ml}$ EP tubes and washed twice with phosphate-buffered saline (PBS) after the animal experiments. We then fixed whole larvae in $4 \%$ paraformaldehyde (PFA) overnight at $4^{\circ} \mathrm{C}$. After the larvae were washed twice with PBS, they were sequentially infiltrated with $20,40,80$ and $100 \%$ propylene glycol at room temperature for $15 \mathrm{~min}$ each and stained with $0.5 \%$ Oil Red O in $100 \%$ propylene glycol at $65^{\circ} \mathrm{C}$ in the dark for $1 \mathrm{~h}$. We washed the stained larvae sequentially with 100 , 80,40 and $20 \%$ propylene glycol for $\sim 20$ min each to fade the background color. Finally, the stained larvae were washed with PBS and stored in $70 \%$ glycerol at $4^{\circ} \mathrm{C}$. The larvae were observed and photographed on a bright-field dissecting microscope (Olympus SZX10; Olympus, Tokyo, Japan).

$H \& E$ staining of paraffin sections. Larvae were fixed in $4 \%$ PFA at $4^{\circ} \mathrm{C}$ overnight and embedded in paraffin according to standard procedures (12). Then, $4 \mu \mathrm{m}$ sections were stained with hematoxylin and eosin (H\&E), and the pathological changes in the liver were observed and photographed using a light microscope (Nikon Eclipse Ni-U; Nikon, Tokyo, Japan).

Nile Red staining. Whole zebrafish Nile Red staining was performed as previously described (13). In total, $0.5 \mathrm{mg}$ of Nile Red was dissolved in $1 \mathrm{ml}$ of acetone, and then diluted with $75 \%$ glycerinum and $25 \%$ water. We washed the fixed larvae 3 times with PBS. The larvae were then permeabilized with $0.1 \%$ Triton in citric acid solution at $65^{\circ} \mathrm{C}$ for $2 \mathrm{~h}$, washed with PBS, and stained with $0.5 \mu \mathrm{g} / \mathrm{ml}$ Nile Red at room temperature in the dark for $50 \mathrm{~min}$. After the larvae were washed with PBS, they were stained with DAPI at room temperature in the dark for $10 \mathrm{~min}$ and then washed 3 times with PBS. Finally, we photographed the stained larvae using a confocal microscope (Nikon C2 Plus).

TUNEL staining. TUNEL assay was performed on paraffinembedded liver sections using an In Situ Cell Death Detection kit, POD (Roche Diagnostics, Basel, Switzerland). Permeabilized larvae were washed with PBS and stained with 1:50 TUNEL at $4^{\circ} \mathrm{C}$ in the dark overnight. The next day, the larvae were stained with DAPI and photographed as mentioned above.

Reverse transcription and quantitative PCR ( $q P C R)$. Total RNA was extracted from 20 larvae that had been smashed with $1 \mathrm{ml}$ syringe pumps, and the total RNA concentrations were measured using a Thermo NanoDrop spectrophotometer. RNA was reverse-transcribed into cDNA using a Takara reverse transcription kit (Takara, Tokyo, Japan). Quantitative PCR was performed using $0.1 \mu \mathrm{mol} / 1$ of gene-specific primers, SYBR-Green SuperMix (Roche) and a LightCycler ${ }^{\circledR} 96$ 


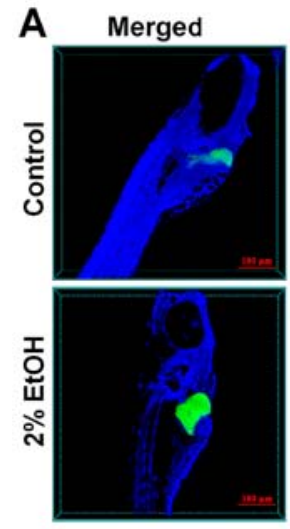

C

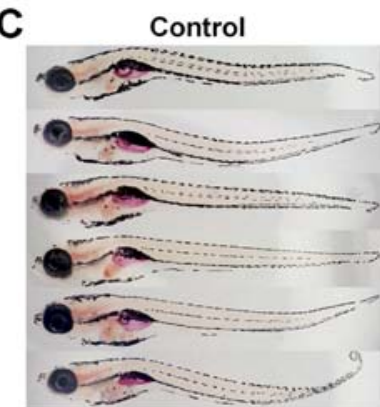

Liver
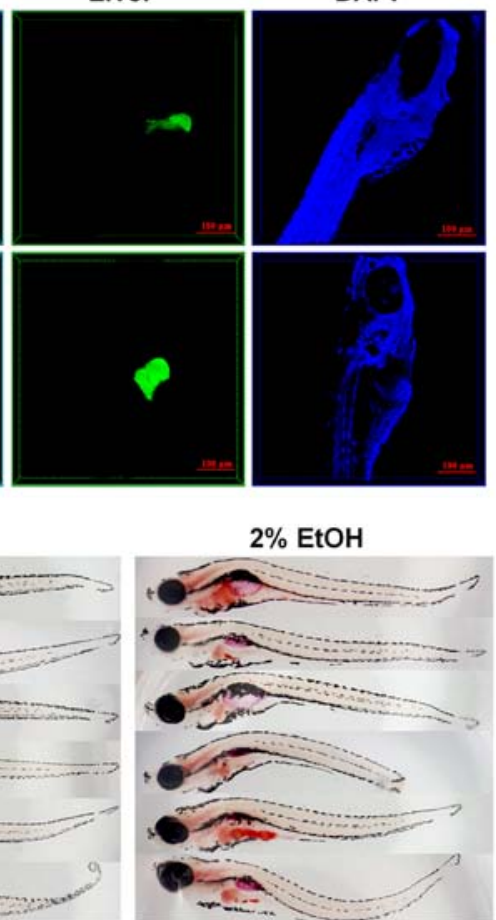

DAPI

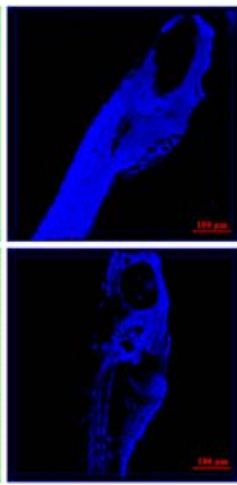

B
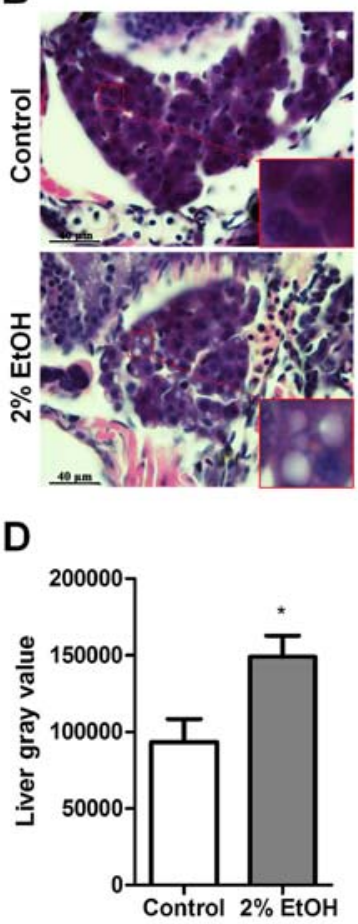

Figure 1. An alcoholic fatty liver zebrafish larvae model was established. (A) In situ morphological changes in the livers of $T g(l f a b p 10 \alpha-e G F P)$ zebrafish larvae subjected to $350 \mathrm{mmol} / 1$ ethanol exposure as detected by confocal microscopy. (B) Representative liver histopathology (H\&E staining) of the livers of WT zebrafish larvae. (C) Oil Red O staining was used to detect hepatic steatosis in response to alcoholic injury in larvae. (D) Quantitative analysis of Oil Red O staining results. The positive staining gray value was calculated using ImageJ software (n=20/group, two experiments). The data are presented as the mean $\pm \mathrm{SEM} ;{ }^{*} \mathrm{P}<0.05$ vs. the control group.

Table I. Primers used to quantify mRNA levels.

\begin{tabular}{|c|c|c|}
\hline Gene & FP sequence $\left(5^{\prime}-3^{\prime}\right)$ & RP sequence $\left(5^{\prime}-3^{\prime}\right)$ \\
\hline rppO & ctgaacatctcgeccttctc & tagccgatctgcagacacac \\
\hline cyp $2 y 3$ & tattcccatgctgcactctg & aggagcgtttacctgcagaa \\
\hline cyp3a65 & aaaccctgatgagcatggac & caagtctttggggatgagga \\
\hline chop & aggaaagtgcaggagctgac & ctccacaagaagaatttcctcc \\
\hline gadd45aa & tggctttgtttgtgggactt & tggaaaacagtccactgaga \\
\hline hmgcra & ctgaggctctggtggacgtg & gatagcagctacgatgttggcg \\
\hline hmgcrb & cctgttagccgtcagtgga & tctttgaccactcgtgccg \\
\hline fasn & gagaaagcttgccaaacagg & gagggtcttgcaggagacag \\
\hline fabp $10 \alpha$ & ttacgctcaggagaactacga & ggatgtgggagaatcggtcag \\
\hline edeml & gacagcagaaaccctcaagc & catggccetcatcttgactt \\
\hline echs1 & agatgcagaatcgaaccttccaa & gagatagcaaactcacatcctccg \\
\hline fads 2 & tcatcgtcgctgttattctgg & tgaagatgttgggtttagcgtg \\
\hline
\end{tabular}

(Roche). The qPCR conditions were as follows: one cycle of $95^{\circ} \mathrm{C}$ for $10 \mathrm{~min}$; followed by 45 cycles of $95^{\circ} \mathrm{C}$ for $10 \mathrm{sec}, 60^{\circ} \mathrm{C}$ for $10 \mathrm{sec}$ and $72^{\circ} \mathrm{C}$ for $10 \mathrm{sec}$; and a final cycle of $95^{\circ} \mathrm{C}$ for $10 \mathrm{sec}, 65^{\circ} \mathrm{C}$ for $60 \mathrm{sec}$ and $97^{\circ} \mathrm{C}$ for $1 \mathrm{sec}$. RppO (ribosomal protein $\mathrm{P} 0$ ) was used as the reference; the primer sequences are listed in Table I. The mRNA level was calculated by the cycle threshold $(\mathrm{Ct})$ method $\left(2^{-\mathrm{Ct}(\text { target })} / 2^{-\mathrm{Ct}(\mathrm{rpp} 0)}\right)$.

Statistical analysis. Statistical analysis was carried out using SPSS 16.0 statistical software (SPSS, Inc., Chicago, IL, USA).
The results are expressed as the mean \pm standard error of the mean (SEM). Statistical analysis was performed using an unpaired t-test or one-way analysis of variance (ANOVA), followed by Tukey's multiple comparison test with dependent experimental designs. One-way ANOVA and t-tests were used for analysis. Differences were considered statistically significance at $\mathrm{P}<0.05$.

\section{Results}

An alcoholic fatty liver zebrafish larva model was established. We chose to expose larvae to ethanol during a window after the liver is formed (96 hpf) and before all the yolk is utilized (5.5-6 dpf) to avoid the metabolic impact of fasting (14). We defined acute exposure as $32 \mathrm{~h}$, which is distinct from the chronic exposure that occurs in alcoholics.

Previous studies indicate that ethanol causes distinct morphological phenotypes, hepatomegaly and behavioral abnormalities in nearly all larvae after $32 \mathrm{~h}$ of exposure to $350 \mathrm{mmol} / \mathrm{l}$ ethanol $(12,15)$. In order to confirm the hepatic morphological phenotypes, we tracked individual $4 \mathrm{dpf}$ $\operatorname{Tg}($ lfabpl0 $\alpha-e G F P)$ larvae exposed to $350 \mathrm{mmol} / \mathrm{l}$ ethanol over $32 \mathrm{~h}$ and verified these findings in clear phase. Hepatomegaly and lordosis were observed in nearly all larvae after $32 \mathrm{~h}$ of ethanol exposure (Fig. 1A and C).

After the larvae were exposed to $350 \mathrm{mmol} / 1$ ethanol for $32 \mathrm{~h}$, serious lipid accumulation was observed in the liver as detected by H\&E staining of the paraffin-embedded sections (Fig. 1B) and Oil Red O staining of whole larvae (Fig. 1C). The quantification of positive Oil Red O staining in the liver was performed 

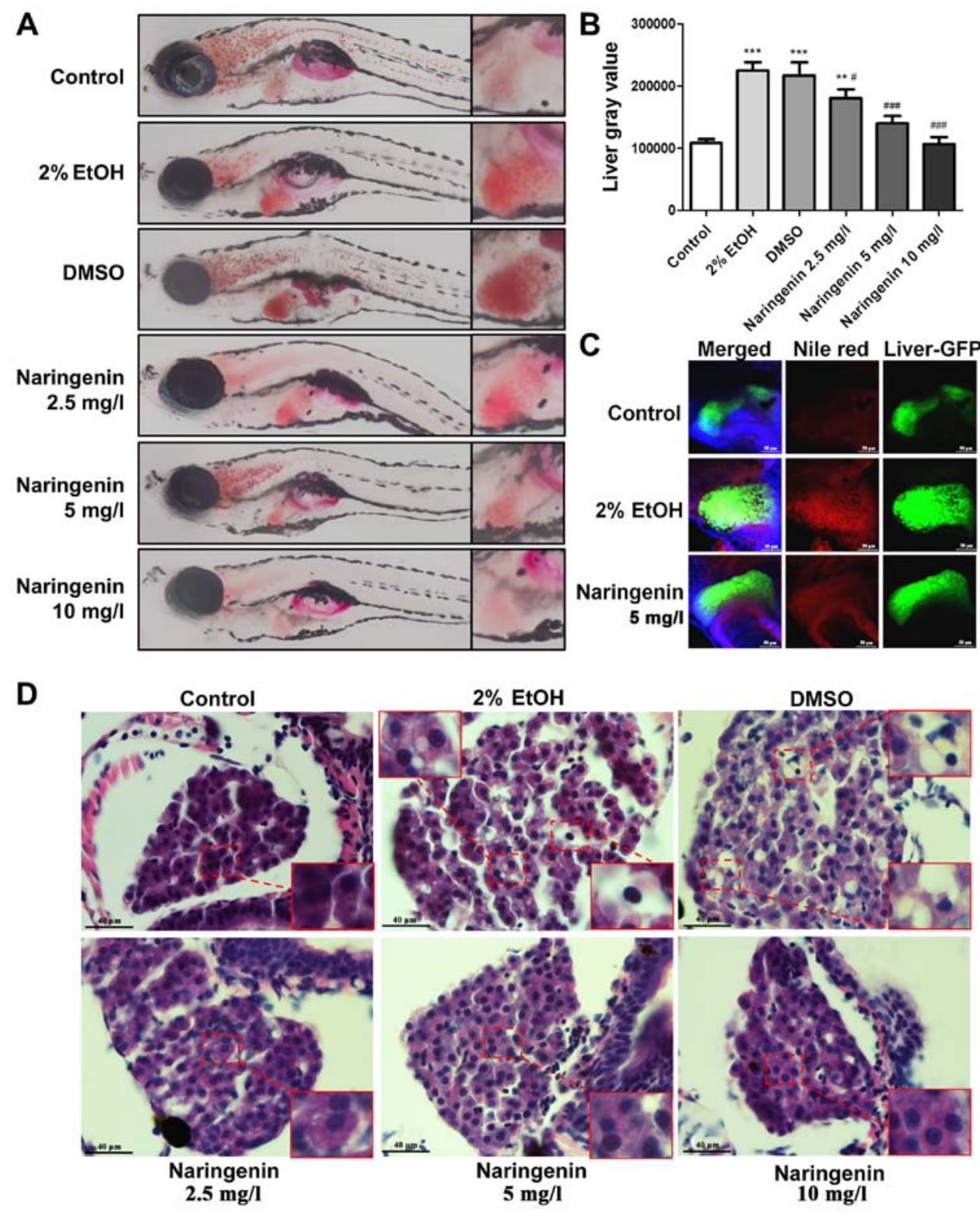

Figure 2. Naringenin reduces alcohol-induced liver steatosis in zebrafish larvae. (A) Oil Red O staining was used to detect liver histopathology and hepatic lipid accumulation in response to naringenin treatment in zebrafish larvae. (B) Quantitative analysis of Oil Red O staining results ( $\mathrm{n}=20 / \mathrm{group}, 3$ experiments). The data are presented as the mean $\pm \mathrm{SEM} ;{ }^{* *} \mathrm{P}<0.01$ vs. control group; ${ }^{* * *} \mathrm{P}<0.001$ vs. control group; ${ }^{*} \mathrm{P}<0.05$ vs. $2 \%$ EtOH group; ${ }^{\# \# "} \mathrm{P}<0.001$ vs. $2 \%$ EtOH group. (C) Selective fluorescent staining (Nile red) for intracellular lipid droplets was used to detect the hepatic response to ethanol exposure with or without naringenin treatment in $T g(l f a b p 10 \alpha-e G F P)$ larvae. (D) H\&E staining was used to detect the changes in liver histopathology in response to naringenin treatment in zebrafish larvae.

using ImageJ software (Fig. 1D), which further confirmed that $32 \mathrm{~h}$ of exposure to $350 \mathrm{mmol} / \mathrm{l}$ ethanol successfully induced hepatic steatosis in zebrafish larvae.

Naringenin reduces alcohol-induced liver steatosis in zebrafish larvae. Although massive lipid deposition was visible in the livers of larvae after ethanol exposure as detected by whole body Oil Red O staining, notably, the obvious hepatic steatosis induced by ethanol exposure was significant dosedependently reduced by naringenin (Fig. 2A). Based on the Oil Red O staining results, hepatic steatosis was quantified in terms of gray value using ImageJ software. The gray value analysis further indicated that naringenin significantly downregulated hepatic steatosis with a dose-dependent change and that $5 \mathrm{mg} / \mathrm{l}$ and $10 \mathrm{mg} / \mathrm{l}$ dosages almost reversed the alcoholic lipid accumulation in larvae (Fig. 2B). In contrast, the selective fluorescent staining (Nile Red) for intracellular lipid droplets was used to detect the hepatic response to ethanol exposure with or without naringenin treatment in $T g(l f a b p 10 \alpha-e G F P)$ larvae. Consistent with the Oil Red O staining results, naringenin treatment $(5 \mathrm{mg} / \mathrm{l}, 48 \mathrm{~h})$ significantly reduced the alcohol-induced lipid droplet accumulation in the livers of exposed larvae (Fig. 2C). Furthermore, the hepatic pathologic change was confirmed by H\&E staining of paraffin-embedded larvae sections (Fig. 2D).

Naringenin treatment improves alcohol metabolism in zebrafish larvae. Cytochrome P450 family 2 subfamily E member 1 (cyp2el), the major enzyme that generates oxidative stress by mediating ethanol metabolism, is proposed to play a 

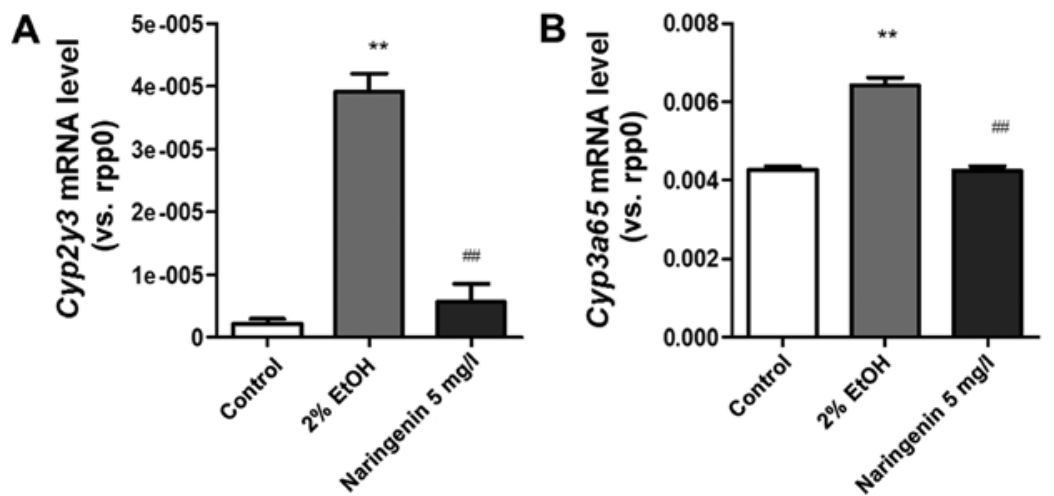

Figure 3. Naringenin treatment improves alcohol metabolism in zebrafish larvae. (A and B) Real-time PCR analysis of the mRNA levels of alcohol metabolism genes (cyp3a65 and cyp2y3) in zebrafish larvae after alcoholic injury with or without naringenin treatment (n=20/group, 3 experiments). The data are presented as the mean $\pm \mathrm{SEM} ;{ }^{* *} \mathrm{P}<0.01$ vs. control group; ${ }^{* \#} \mathrm{P}<0.01$ vs. $2 \%$ EtOH group.
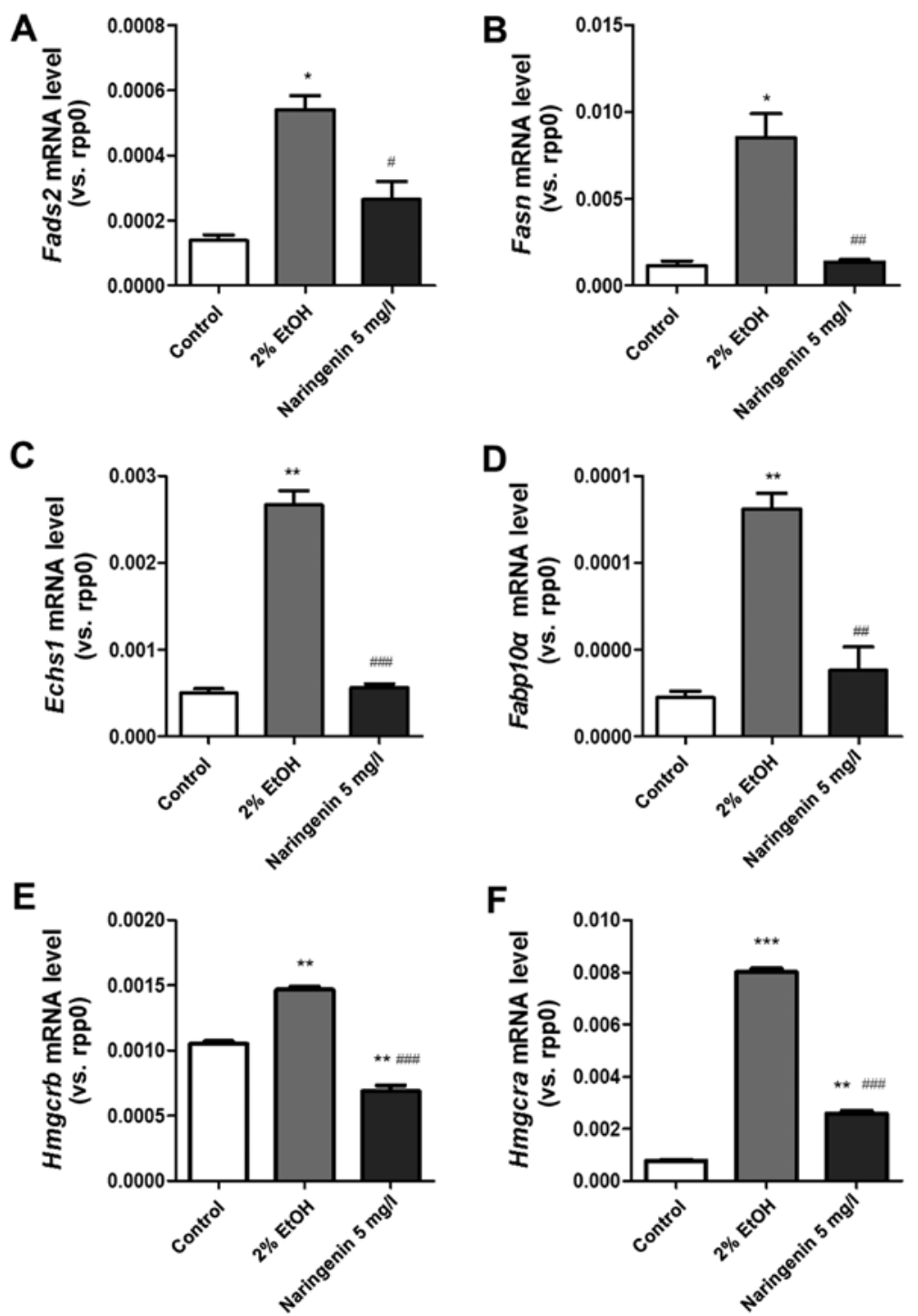

Figure 4. Naringenin treatment improves lipid metabolism and protects zebrafish larvae against alcoholic injury. (A-F) Real-time PCR analysis of the mRNA levels of lipid metabolism genes in zebrafish larvae after alcoholic injury with or without naringenin treatment ( $\mathrm{n}=20 / \mathrm{group}, 3$ experiments). The data are presented as the mean $\pm \mathrm{SEM} ;{ }^{*} \mathrm{P}<0.05$ vs. control group; ${ }^{* *} \mathrm{P}<0.01$ vs. control group; ${ }^{* * *} \mathrm{P}<0.001$ vs. control group; ${ }^{\text {}} \mathrm{P}<0.05$ vs. $2 \%$ EtOH group; ${ }^{\# \#} \mathrm{P}<0.01$ vs. $2 \%$ EtOH group; ${ }^{\# \#} \mathrm{P}<0.001$ vs. $2 \%$ EtOH group.

major role in ALD pathology in mammals. Cytochrome P450, family 2, subfamily Y, polypeptide 3 (cyp2y3) and cyp2e1 are homologous genes that are critical for ethanol metabolism in the zebrafish liver (14). Upregulation of cyp $2 y 3$ accelerates the 
A

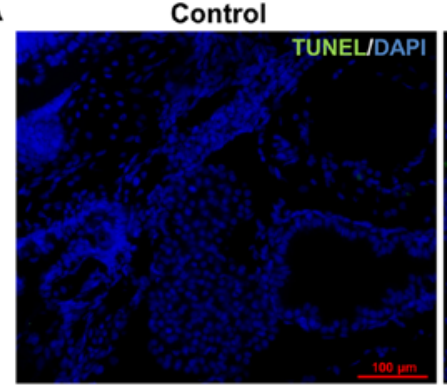

B

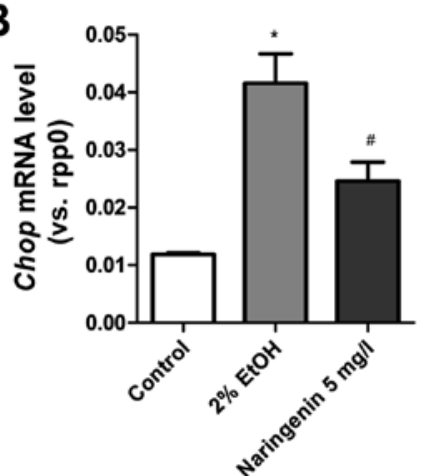

$2 \%$ EtOH
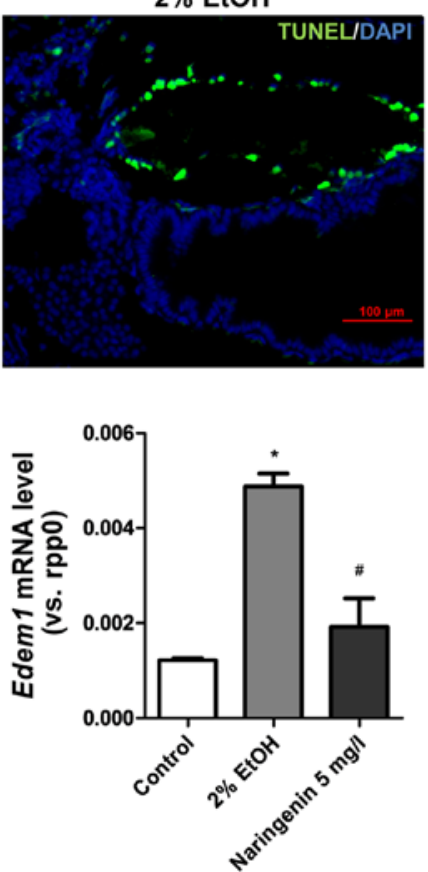

Naringenin $5 \mathrm{mg} / \mathrm{l}$
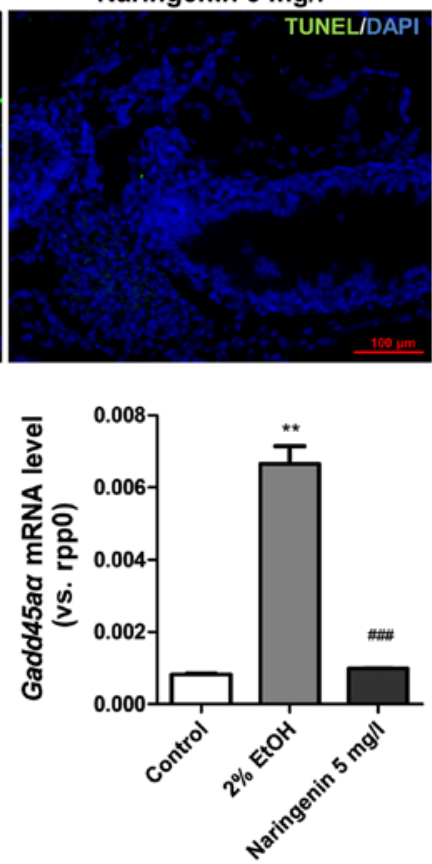

Figure 5. Naringenin attenuates endoplasmic reticulum stress and DNA damage in zebrafish larvae with alcoholic injury. (A) In situ detection of cell apoptosis by TUNEL staining in paraffin-embedded sections of zebrafish larvae after ethanol exposure with or without naringenin treatment. (B) Real-time PCR analysis of the mRNA levels of endoplasmic reticulum stress- and DNA damage-related genes (chop, gadd45 $\alpha$ a and edem1) in larvae (n=20/group, 3 experiments). The data are presented as the mean $\pm \mathrm{SEM} ;{ }^{*} \mathrm{P}<0.05$ vs. control group; ${ }^{* *} \mathrm{P}<0.01$ vs. control group; ${ }^{\#} \mathrm{P}<0.05 \mathrm{vs} .2 \%$ EtOH group; ${ }^{\# \# \#} \mathrm{P}<0.001 \mathrm{vs} .2 \%$ EtOH group.

speed of alcohol metabolism and accumulation of acetaldehyde, which increase the quantity of liver damage (14). Our data indicated that the mRNA level of cyp2y3 was significantly elevated in zebrafish larvae after alcohol exposure compared to the control group, while naringenin treatment reversed this effect on the cyp2y3 mRNA level (Fig. 3A). Similar to cytochrome P450, family 3, subfamily A, polypeptide 65 (cyp3a65) (Fig. 3B), the ortholog of cytochrome P450, family 3 , subfamily A (cyp3a) is also mainly expressed in the liver and plays a key role in the metabolism of endogenous substrates and xenobiotics (16). The beneficial effects of naringenin treatment may be due to the improvement of alcohol metabolism and reduction of toxic substances.

Naringenin treatment improves lipid metabolism and protects zebrafish larvae against alcoholic injury. We next evaluated whether naringenin could attenuate lipid metabolism, improve lipid homeostasis and prevent alcohol-induced hepatic steatosis. The key genes ( fads2, fasn, echs $1, f a b p 10 \alpha$, hmgcra and hmg crb) involved in lipid metabolism were chosen; these genes are related to cholesterol biosynthesis, fatty acid synthase and desaturase, and mitochondrial enzyme (17-21). Quantitative PCR results (Fig. 4A-F) indicated that alcoholic treatment induced significant upregulation of zebrafish larva mRNA (fads2, fasn, echs 1, fabpl0 $\alpha$, hmgcra and hmgcrb), which was reversed by naringenin treatment.

Naringenin attenuates endoplasmic reticulum stress and DNA damage in zebrafish larvae with alcoholic injury. Endoplasmic reticulum stress and DNA damage play critical roles in the pathogenesis of alcoholic liver injury $(14,22)$. We examined the anti-apoptotic effects of naringenin on

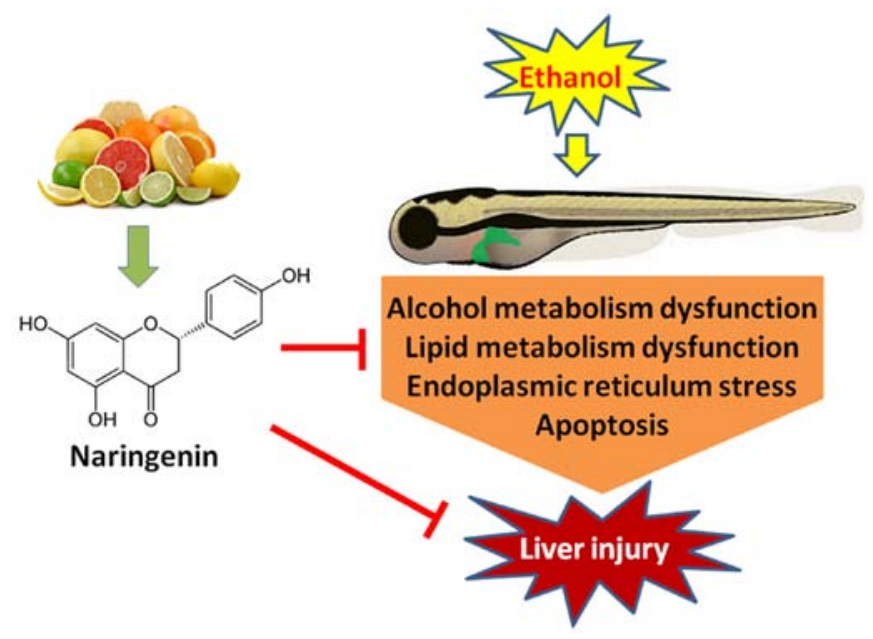

Figure 6. Graphical illustration. Naringenin inhibits alcohol-induced liver steatosis and injury in zebrafish larvae by reducing apoptosis and DNA damage and harmonizing alcohol and lipid metabolism.

alcohol-induced liver steatosis by TUNEL staining and compared the results with mRNA levels of DNA-damage inducible transcript 3 (chop) (26) and growth arrest and DNA damage-inducible, $\alpha$, a $(\operatorname{gadd} 45 \alpha a)(24)$, which are critical biomarkers of endoplasmic reticulum stress and DNA damage. TUNEL staining indicated that naringenin significantly reduced hepatocyte apoptosis in zebrafish larvae with alcoholic injury (Fig. 5A). The detection of chop and gadd45 $\mathrm{\alpha a}$ mRNA levels also indicated that naringenin reversed the significantly increased mRNA levels of chop and gadd45 $\alpha a$ after alcohol treatment (Fig. 5B). Thus, naringenin 
inhibited endoplasmic reticulum stress and DNA damage and reduced ethanol-induced cell apoptosis in zebrafish larvae.

\section{Discussion}

Hepatic steatosis is the first response to alcohol abuse (2) and may progress to more severe liver diseases. Chronic steatosis is a prerequisite for developing steatohepatitis and cirrhosis and makes hepatocytes susceptible to damage (25). Therefore, inhibiting hepatic lipid accumulation during alcohol exposure may stop further liver damage. Naringenin acts in a manner similar to metformin to reduce hepatic glucose production in hepatocytes (7). In addition, naringenin was found to improve various aspects of lipid homeostasis and to mitigate adipose tissue inflammation in vivo (6,7,26-28). However, the effects of naringenin on alcoholic and metabolic abnormalities have not been studied. The present study is the first to use alcoholexposed zebrafish to examine the effects of naringenin on ethanol metabolism and major aspects of ALD pathology, including steatosis, ER stress and apoptosis, in zebrafish larvae. In the present study, we confirmed and expanded on previous findings $(12,15)$ that acute exposure of zebrafish larvae to $350 \mathrm{mmol} / \mathrm{l}$ ethanol was suitable for establishing an alcoholic fatty liver zebrafish model. Furthermore, we demonstrated that naringenin treatment could prevent acute alcohol-induced hepatic steatosis, dyslipidemia, and cell death. Naringenin markedly attenuated alcohol and lipid metabolism and hepatic lipid accumulation, collectively resulting in the attenuation of steatosis.

The alcoholic fatty liver zebrafish larvae model is easily and quickly generated. Obtaining liver tissue and blood from zebrafish larvae is difficult and prevents the detection of mRNA and protein expressed in the liver and the measurement of biochemical parameters related to liver function; however, zebrafish larvae have other advantages, including their short breeding cycle and transparent soma. We can obtain vast larvae in a short time, and it is easy to observe whole body staining of larvae.

In the present study, we first detected the anti-steatosis effect of naringenin against ethanol exposure in zebrafish larvae. Both Oil Red O staining and H\&E pathological staining results showed that naringenin reduced alcoholic liver steatosis in zebrafish larvae and that the therapeutic effects had a dose-dependent tendency with the minimum and optimum naringenin concentration $(5 \mathrm{mg} / \mathrm{l})$. After we confirmed the anti-steatosis effect of naringenin, we examined the potential role of naringenin in alcohol-induced cell death and injury. TUNEL staining showed that apoptosis may contribute to alcoholic liver injury. chop and gadd $45 \alpha a$ are growth arrest and DNA damage genes, respectively. chop regulates the transcription of lipid metabolism genes (29), and upregulation of the chop protein causes lipid metabolism disorders in the liver (23). chop is also known as a specific transcription factor of endoplasmic reticulum stress. In contrast, ER degradation-enhancing $\alpha$-mannosidase-like protein 1 (edeml), a key gene involved in the unfolded protein response, was increased significantly in patients with steatosis (30). Increased mRNA expressions of chop, gadd $45 \alpha a$ and edeml after ethanol exposure indicated that endoplasmic reticulum stress and DNA damage were more serious. In contrast, less apoptosis and downregulation of chop, gadd45 $\alpha a$ and edem 1 mRNA levels were induced by naringenin treatment. Therefore, we confirmed that naringenin could suppress alcohol-induced steatosis and damage in zebrafish larvae.

HMG-CoA reductases, including HMG coenzyme A reductase a (hmgcra) and HMG coenzyme A reductase b (hmgcrb), are key enzymes in lipid metabolism and mainly regulate cholesterol biosynthesis genes $(17,31)$. Fatty acid synthase (fasn) regulates the synthesis and desaturation of fatty acids (19). Fatty acid desaturase 2 (fads2) is a dyslipidemia-related gene that mainly metabolizes unsaturated fatty acids, is involved in glucose metabolism and influences the concentrations of total cholesterol, low-density lipoprotein cholesterol, high-density lipoprotein cholesterol and triglycerides (18). Fatty acid-binding protein 10a $(f a b p 10 \alpha)$ is an intracellular fatty acid-binding protein that is involved in intracellular lipid metabolism and fatty acid transport (21). Enoyl-CoA hydratase, short chain 1 (echs1), a gene that encodes a mitochondrial enzyme, is related to the degradation of amino acids and essential fatty acids, and echs1 mutations cause severe metabolic disorders (20). The fads2, fasn, echs1, fabp10 $\alpha$, hmgcra and hmgcrb genes, which regulate lipid metabolism, were significantly upregulated by ethanol exposure, thus indicating that alcohol exposure caused disordered lipid metabolism in larvae. Naringenin treatment significantly improved lipid metabolism by reducing fads 2 , fasn, echsl, fabp10 $\alpha$, hmgcra and hmgcrb mRNA levels.

In contrast, cyp $2 y 3$ and cyp $3 a 65$, the orthologs of cytochrome P450 CYP2 (cyp2) and cyp3a, are required for ethanol metabolism mainly in zebrafish liver. In zebrafish, the closest cyp2e1 homolog is cyp2y3, which is $43 \%$ identical to the human protein (14). Blocking cyp2 homologs significantly reduces alcohol metabolism and oxidative stress. In addition, cyp $3 a 65$ plays a critical role in the metabolism of endogenous substrates and xenobiotics (16). The roles of cyp2y3 and cyp2el in biological disturbances are emphasized in the initiation and progression of fatty liver disease caused by alcohol consumption (14). Notably, our data indicated that naringenin interruption could significantly downregulate the elevated mRNA levels of cyp $2 y 3$ and cyp $3 a 65$ in zebrafish larvae, which were induced by ethanol exposure. The beneficial effects of naringenin treatment may be due to the improvement of alcohol metabolism and reduction of toxic substances. Combined with the above observations, we realized that alcoholic injury in zebrafish larvae mainly resulted from disordered lipid and alcohol metabolism and that naringenin could improve the inordinate metabolism to inhibit alcoholic steatosis and injury.

Finally, we summarize the critical roles of naringenin in inhibiting alcohol-induced liver steatosis as showed in a graphical illustration (Fig. 6). The present study demonstrated that naringenin inhibits alcohol-induced liver steatosis and injury in zebrafish larvae by reducing apoptosis and DNA damage and harmonizing alcohol and lipid metabolism. Further experimentation is needed to illuminate the pathway by which apoptosis is reduced and alcohol and lipid metabolism is balanced. Naringenin readily accumulates in plasma after ingesting orange juice, grapefruit juice and tomato paste or sauce (5), suggesting the bioavailability of naringenin in individuals who consume naringenin food sources regularly. 
Further pre-clinical and human intervention studies may help to determine whether naringenin can protect against alcohol liver diseases and lipid metabolic syndrome in humans.

\section{Acknowledgements}

The present study was supported by the National Natural Science Foundation of China (nos. 81603501 and 81302948), the China Postdoctoral Science Foundation (2016M592508), the Science and Technology Planning Project of Guangdong Province (2014A020221097), the Administration of Traditional Chinese Medicine of Guangdong Province (20162087), the Science and Technology Planning Project of Guangzhou City (201508020014), and the Scientific Research Initiative Program of Southern Medical University (LX2015N003).

\section{References}

1. Ni HM, Bhakta A, Wang S, Li Z, Manley S, Huang H, Copple B and Ding WX: Role of hypoxia inducing factor-1 $\beta$ in alcohol-induced autophagy, steatosis and liver injury in mice. PLoS One 9: e115849, 2014.

2. Orman ES, Odena G and Bataller R: Alcoholic liver disease: Pathogenesis, management, and novel targets for therapy. J Gastroenterol Hepatol 28 (Suppl 1): S77-S84, 2013.

3. Akhlaghi M: Non-alcoholic fatty liver disease: Beneficial effects of flavonoids. Phytother Res 30: 1559-1571, 2016.

4. Moon PD, Choi IH and Kim HM: Naringenin suppresses the production of thymic stromal lymphopoietin through the blockade of RIP2 and caspase-1 signal cascade in mast cells. Eur J Pharmacol 671: 128-132, 2011.

5. Ke JY, Kliewer KL, Hamad EM, Cole RM, Powell KA, Andridge RR, Straka SR, Yee LD and Belury MA: The flavonoid, naringenin, decreases adipose tissue mass and attenuates ovariectomy-associated metabolic disturbances in mice. Nutr Metab 12: 1, 2015

6. Mulvihill EE, Allister EM, Sutherland BG, Telford DE, Sawyez CG, Edwards JY, Markle JM, Hegele RA and Huff MW: Naringenin prevents dyslipidemia, apolipoprotein B overproduction, and hyperinsulinemia in LDL receptor-null mice with diet-induced insulin resistance. Diabetes 58: 2198-2210, 2009.

7. Purushotham A, Tian M and Belury MA: The citrus fruit flavonoid naringenin suppresses hepatic glucose production from Fao hepatoma cells. Mol Nutr Food Res 53: 300-307, 2009.

8. Chtourou Y, Fetoui H, Jemai R, Ben Slima A, Makni M and Gdoura R: Naringenin reduces cholesterol-induced hepatic inflammation in rats by modulating matrix metalloproteinases-2, 9 via inhibition of nuclear factor $\kappa \mathrm{B}$ pathway. Eur $\mathrm{J}$ Pharmacol 746: 96-105, 2015.

9. Zar Kalai F, Han J, Ksouri R, El Omri A, Abdelly C and Isoda H: Antiobesity effects of an edible halophyte Nitraria retusa Forssk in 3T3-L1 preadipocyte differentiation and in $\mathrm{C} 57 \mathrm{~B} 6 \mathrm{~J} / \mathrm{L}$ mice fed a high fat diet-induced obesity. Evid Based Complement Alternat Med 2013: 368658, 2013.

10. Howarth DL, Yin C, Yeh K and Sadler KC: Defining hepatic dysfunction parameters in two models of fatty liver disease in zebrafish larvae. Zebrafish 10: 199-210, 2013.

11. Dai W, Wang K, Zheng X, Chen X, Zhang W, Zhang Y, Hou J and Liu L: High fat plus high cholesterol diet lead to hepatic steatosis in zebrafish larvae: A novel model for screening anti-hepatic steatosis drugs. Nutr Metab 12: 42, 2015.

12. Passeri MJ, Cinaroglu A, Gao C and Sadler KC: Hepatic steatosis in response to acute alcohol exposure in zebrafish requires sterol regulatory element binding protein activation. Hepatology 49: 443-452, 2009.

13. Greenspan P, Mayer EP and Fowler SD: Nile red: A selective fluorescent stain for intracellular lipid droplets. J Cell Biol 100: 965-973, 1985.
14. Tsedensodnom O, Vacaru AM, Howarth DL, Yin C and Sadler KC: Ethanol metabolism and oxidative stress are required for unfolded protein response activation and steatosis in zebrafish with alcoholic liver disease, pp1213-1226, 2013.

15. Howarth DL, Passeri M and Sadler KC: Drinks like a fish: Using zebrafish to understand alcoholic liver disease. Alcohol Clin Exp Res 35: 826-829, 2011.

16. Tseng $\mathrm{H}$, Hseu T, Buhler D, Wang $\mathrm{W}$ and $\mathrm{Hu} \mathrm{C}$ : Constitutive and xenobiotics-induced expression of a novel CYP3A gene from zebrafish larva. Toxicol Appl Pharmacol 205: 247-258, 2005.

17. Suganya S, Nandagopal B and Anbarasu A: Natural inhibitors of HMG CoA reductase - an in silico approach through molecular docking and simulation studies. J Cell Biochem 99: 1-6, 2016.

18. Tian Y, Zhang W, Zhao S, Sun Y, Bian Y, Chen T, Du Y, Zhang J, Wang Z, Huang T, et al: FADSI-FADS2 gene cluster confers risk to polycystic ovary syndrome. Sci Rep 6: 21195, 2016.

19. Zappaterra M, Deserti M, Mazza R, Braglia S, Zambonelli P and Davoli R: A gene and protein expression study on four porcine genes related to intramuscular fat deposition. Meat Sci 121: 27-32, 2016.

20. Olgiati S, Skorvanek M, Quadri M, Minneboo M, Graafland J, Breedveld GJ, Bonte R, Ozgur Z, van den Hout MC, Schoonderwoerd K, et al: Paroxysmal exercise-induced dystonia within the phenotypic spectrum of ECHS1 deficiency. Mov Disord 31: 1041-1048, 2016.

21. Storch J and Thumser AE: The fatty acid transport function of fatty acid-binding proteins. Biochim Biophys Acta 1486: 28-44, 2000.

22. Ren Z, Wang X, Xu M, Yang F, Frank JA, Ke ZJ and Luo J: Binge ethanol exposure causes endoplasmic reticulum stress, oxidative stress and tissue injury in the pancreas. Oncotarget 7: 54303-54316, 2016.

23. Zheng X, Dai W, Chen X, Wang K, Zhang W, Liu L and Hou J: Caffeine reduces hepatic lipid accumulation through regulation of lipogenesis and ER stress in zebrafish larvae. J Biomed Sci 22: 105,2015

24. Zhao XX, Zhang YB, Ni PL, Wu ZL, Yan YC and Li YP: Protein arginine methyltransferase 6 (Prmt6) is essential for early zebrafish development through the direct suppression of gadd45 $\alpha a$ stress sensor gene. J Biol Chem 291: 402-412, 2016.

25. Howarth DL, Lindtner C, Vacaru AM, Sachidanandam R, Tsedensodnom O, Vasilkova T, Buettner C and Sadler KC: Activating transcription factor 6 is necessary and sufficient for alcoholic fatty liver disease in zebrafish. PLoS Genet 10: e1004335, 2014.

26. Cho KW, Kim YO, Andrade JE, Burgess JR and Kim YC: Dietary naringenin increases hepatic peroxisome proliferators-activated receptor $\alpha$ protein expression and decreases plasma triglyceride and adiposity in rats. Eur J Nutr 50: 81-88, 2011.

27. Goldwasser J, Cohen PY, Yang E, Balaguer P, Yarmush ML and Nahmias Y: Transcriptional regulation of human and rat hepatic lipid metabolism by the grapefruit flavonoid naringenin: Role of PPARalpha, PPARgamma and LXRalpha. PLoS One 5: e12399, 2010.

28. Alam MA, Subhan N, Rahman MM, Uddin SJ, Reza HM and Sarker SD: Effect of citrus flavonoids, naringin and naringenin, on metabolic syndrome and their mechanisms of action. Adv Nutr 5: 404-417, 2014

29. Rahman K, Liu Y, Kumar P, Smith T, Thorn NE, Farris AB and Anania FA: C/EBP homologous protein modulates liraglutidemediated attenuation of non-alcoholic steatohepatitis. Lab Invest 96: 895-908, 2016.

30. Congiu M, Ryan MC and Desmond PV: No increase in the expression of key unfolded protein response genes in HCV genotype 3 patients with severe steatosis. Virus Res 160: 420-423, 2011.

31. Cocci P, Mosconi G and Palermo FA: Partial cloning, tissue distribution and effects of epigallocatechin gallate on hepatic 3-hydroxy-3-methylglutaryl-CoA reductase mRNA transcripts in goldfish (Carassius auratus). Gene 545: 220-225, 2014. 